\title{
Calculation method of carbon emission flow in power system based on the theory of power flow calculation
}

ChenSongsong ${ }^{1, a}$, Sun Beibei ${ }^{2, ~ b}$, HeZiheng $^{3, c^{*}}$, WuZhou ${ }^{4, d}$

${ }^{1}$ China Electric Power Research Institute, China

${ }^{2}$ State Grid Jibei Electric Power Company Limited, China

${ }^{3}$ School of Electrical and Electronic Engineering, North China Electric Power University, China
${ }^{4}$ State Grid Anhui Electric Power Energy Saving Service Co.Ltd, China

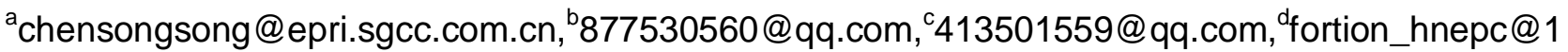

63.com

Keywords: Carbon emission flow, computation, power flow, electric system

Abstract. Carbon emissions' measure and share is basis of many low carbon electric technologies, only the carbon emissions of every participant is specific can the enthusiasm of taking part in electric system emission reduction be motivated. In this article, a calculate method of electric system carbon flow is designed, which used the adjacent properties of carbon emission flow calculate model, and adopted a kind of distributed calculate mode. In this method, by iterative computations of each computation unit located on electric generator port and bus-bar of power grid, the distribution of carbon emission flow in electric system is acquired.

\section{Introduction}

The energy crisis and global climate change has caused wide attention.Reducing the excessive consumption of fossil fuelsand achieving low carbon development has become the common goal of various industries and various countries.Power industry, as an important part of the energy supply, is also the main industry of fossil fuels consumption, causing a lot of carbon emissions.Due to the power structure of our country, the phenomenon is especially remarkable in China.According to statistics, in recent years, China's power industry emissions of carbon dioxide occupies half of the country's carbon emissions caused by fossil fuel combustion.Study low carbon power technology and reduce the carbon emissions of power system is very important for the realization of China's energy-saving emission reduction targets and the promotion of the sustainable development of the whole society ${ }^{[1,2]}$.

The measurement and allocation of carbon emissions is the basis of many low carbon power technology $y^{[3]}$, only the carbon emissions of every participant is specific can the enthusiasm of taking part in electric system emission reduction be motivated. In the past, the measurement methods of carbon emissions are based on the macroscopic data, based on the consumption of fossil energy and other data to statistical calculate direct carbon emissions. It is the measurement method based on consumer side. Releasing carbon emission flow, combining the characteristics of the power system and the development of low carbon concept, establishes a model sharing the responsibility for carbon emissions of electric power system from the perspective of load, namely allocating the carbon emissions in the process of generating to each user according to the users electricity and power flow tracing. Carbon emission flow model connects the carbon emissions with 
the interests subject of electric energy, making the carbon emission reduction responsibility sharing more equitable, helping to eliminate the phenomenon of carbon leakage and stimulating the user side to participate in the power system carbon emissions reduction ${ }^{[4]}$.

According to the published papers, the carbon emission flow is dependent on the power flow and the virtual network flow which is used to characterize the carbon emissions maintaining any branch flow in the power system ${ }^{[5]}$. Carbon emission flow of power system is equivalent to the label of carbon emissions on each branch.In power systems, carbon emission flow starts from power plants (power plant nodes), entering the power system with the power plant, flowing in the grid with the power flow in the system, and ultimately flows into the consumer terminal (load node) of the consumer side, as shown in Fig. 1.

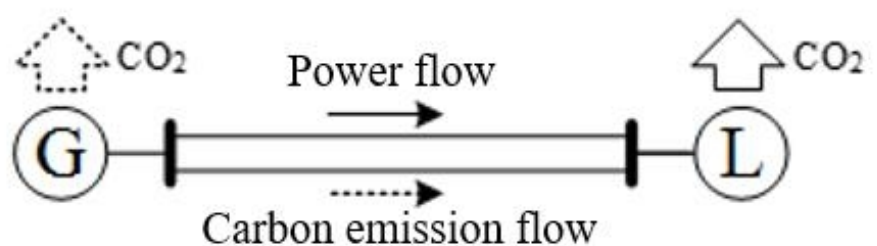

Fig. 1. Carbon emission flow of power system

The existingmethodisacentralized algorithm ${ }^{[6]}$, which is based on the control of the generatorsoutput and carbon emission densityof the whole system, the active power flow and grid structureof all lines and the electric data of all load nodes, and unified computing by a central server.This method needs to establish a data collection system which covers the whole system in central server, and it will increase the burden of the central server and is difficult to achieve. To this end, a distributed calculating method for carbon emission flow is presented in this paper ${ }^{[7-9]}$.

\section{Summary}

In this method, the distributed computing idea is adopted, which is based on the computing unit of the electrical equipment, and the distribution of the whole system's carbon emission flow is obtained by the iterative method.Main steps: One is the installing of calculation unit on all devices which are related to the carbon emission flow distribution. The unit collects the information of the local active power flow and the direct carbon emissions. On the other hand, it's in the iterative communication with the adjacent computing units involving in the calculation of the total system carbon emissions. The other is based on the characteristics of that carbon emission index of each node only adjacentindex value of the related nodes. It's boundary conditions have been confirmed by each generation unit of carbon emissions density and output parameters in the system, through the iterative calculation and communication of adjacent calculation units, from the near to the distant, and gradually calculates carbon releasing index of all nodes which are connected with the electric generator in the whole system.

Power system carbon emission flow's basic index mainly includes branch carbon flow, branch carbon flow rate, branch carbon flow density and node carbon potential.In the case of known system active power flow distribution, the carbon flow rate of the branch and the branch flow rate can be calculated by the carbon potential of the node.

For exit nodeof generator, the node carbon potential is equal to the carbon emission densityofgenerating units. Because the operating characteristics of the unit can be determined, it belongs to the known boundary conditionsin the calculation of carbon emission flow. 


$$
e_{b}^{B}=\frac{\sum_{l \in N^{+}} P_{l} \cdot \rho_{l}}{\sum_{l \in N^{+}} P_{l}}
$$

In Eq. $1, N^{+}$is the collection of branch that all the branch connecting node B flowing into node $\mathrm{B}, l$ is branch number, $P_{l}$ is active power flow of branch $l$, and $\rho_{l}$ is branch carbon flow density of branch $l$.

The carbon flow density of each branch is equal to the carbon potential of the node power flowoutflownode. Namely,there is the following formula:

$$
\rho_{l^{-}}=e_{b}^{B} \quad \forall l^{-} \in N L_{b}^{-}
$$

In Eq. 2,N $L_{b}^{-}$is the collectionof all the branches whichhave flow flowing from the node B.

The Eq. 1 and Eq. 2 indicate that, for one node, if all the nodes of the neighboring nodes whose active power are injected into a node are known, then the carbon potential of this node can be calculated.The node carbon potential of the adjacent nodes can be obtained by the communication between the nodes, and the active power can be obtained by local measurement.

\section{Method design}

Distributedcalculation of carbon emission flow

In order to achieve distributed calculation of carbon emission flow, which need to install computation units on the nodes of electric system relating to the carbon flow distribution, including the generators, bus-bar of different voltage grade in the power grid and electricity load, etc. Each node should with specific serial number, keeping the node number that exists connection relations with the current node in the computation units of each node, in order to facilitate the communication among the computation units of all the nodes.

The computation units installed on the generator set need to measure the carbon emissions of the generator set. This parameter is the given boundary condition, which can be inputted to the computation units of generator set by the other methods, such as the state monitor of boiler,chimney flue gas continuous monitoring system and so on.

All the computation units need to measure the flow and size of local active power direction and change the computing information with the "adjacent" computation unit. The adjacent nodes here refer that the power flow of two nodes has the strict equivalence relation of electrical flow. A, B, C can be counted as "adjacent" node in Fig. 2, because they have the strict equivalence relations of electrical flow $P_{A B}=P_{B C}$. At this point, the node $\mathrm{B}$ can get rid of the computation unit and node $\mathrm{A}$ and node $\mathrm{C}$ can communicate with each other directly. Node $\mathrm{C}$ with Node $\mathrm{D}$ can be counted as "adjacent" nodes, node $\mathrm{C}$ with Node $\mathrm{E}$ also can be counted as "adjacent" nodes, so it needs to establish mutual communication between them. Between node B with node $\mathrm{D}$ and between node $\mathrm{B}$ with node E,it can't be counted as "adjacent" nodes, because $P_{B C} \neq P_{C D}, P_{B C} \neq P_{C E}$."Unadjacent" between nodes don't need the iterative calculation of carbon flow. 


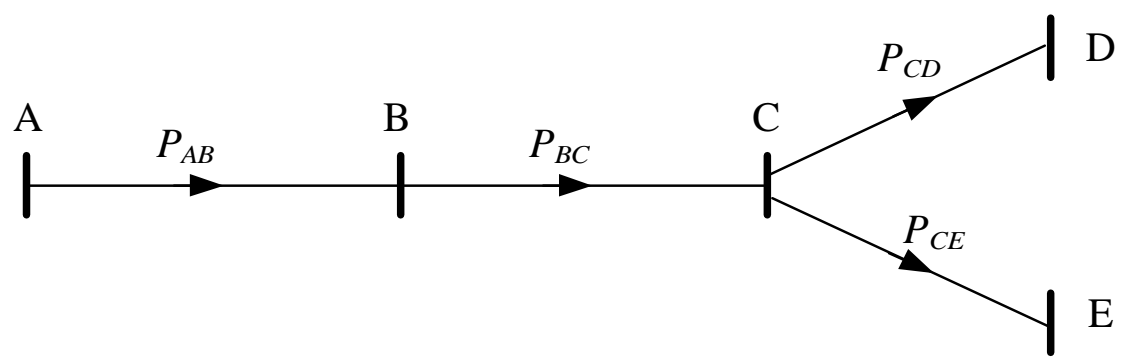

Fig. 2 "Adjacent" nodes in the calculation of carbon emissions flow

Communication between each computation unit has no specific restriction, can making full use of communication infrastructure in electric system. The computation unit's coordinate calculation need theunified clock signal.Exchanging an information interaction between the adjacent computation units cell under each iteration calculation, and according to the gained information calculatethe related indicators of local carbon flow.Each interactive information between adjacent computation unitsshould includenode carbon potential and clock labels of the node at least.

"Pass" calculation of distributedcomputation unit

With the support of the basic computation units, the "pass" calculation of carbon emission flow can be shown through radial system in Fig. 3.

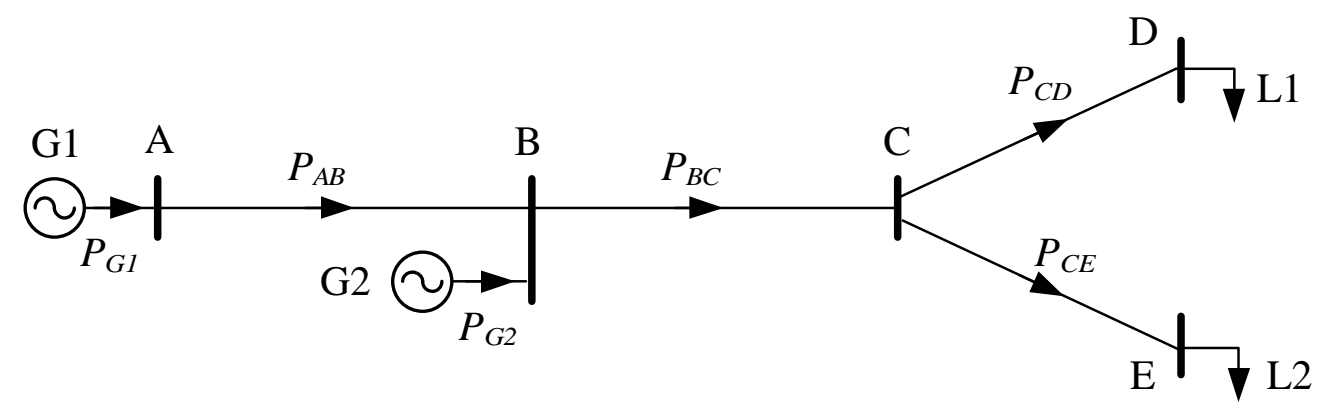

Fig. 3The "pass" calculation of distributedcomputation unit

Computation unitsof G1, G2obtaincarbon density of two sets by an external device, can be seem as its carbon potential. Moments later, the iterative calculation process of the system is as follows:

$\ddot{y}$ The initial conditions: node carbon potentials of G1 and G2 are known.

\begin{tabular}{|c|c|c|c|c|c|c|c|}
\hline & G1 & G2 & A & B & C & D & E \\
\hline $\begin{array}{c}\text { Node } \\
\text { carbon } \\
\text { potential }\end{array}$ & known & known & unknown & unknown & unknown & unknown & unknown \\
\hline
\end{tabular}

$\ddot{y}$ The first iteration: The computation unit of node A obtainthe carbon potential of node Aby communicatingwith the computation unit of generator G1. In the initial conditions, the carbon potential of node $\mathrm{A}$ is unknown and the active power of node $\mathrm{A}$ into the node $\mathrm{B}$, so the carbon potential of node B can't calculate in the first iteration. The carbon potential of other nodes can't befigured out in the current iterationin a similar way.

\begin{tabular}{|c|c|c|c|c|c|c|c|}
\hline & G1 & G2 & A & B & C & D & E \\
\hline $\begin{array}{c}\text { Node } \\
\text { carbon } \\
\text { potential }\end{array}$ & known & known & known & unknown & unknown & unknown & unknown \\
\hline
\end{tabular}


$\ddot{y}$ The second iteration: Node B obtain the carbon potential of node A and generator G2by the information interaction, the active power is into PAB and PG2, the carbon potential of node B can be obtained through the Eq. 1.

\begin{tabular}{|c|c|c|c|c|c|c|c|}
\hline & G1 & G2 & A & B & C & D & E \\
\hline $\begin{array}{c}\text { Node } \\
\text { carbon } \\
\text { potential }\end{array}$ & known & known & known & known & unknown & unknown & unknown \\
\hline
\end{tabular}

$\ddot{y}$ The third iteration: The carbon potential of node $C$ can be obtained through communicating with node B.

\begin{tabular}{|c|c|c|c|c|c|c|c|}
\hline & G1 & G2 & A & B & C & D & E \\
\hline $\begin{array}{c}\text { Node } \\
\text { carbon } \\
\text { potential }\end{array}$ & known & known & known & known & known & unknown & unknown \\
\hline
\end{tabular}

$\ddot{y}$ The fourth iteration: The carbon potential of node D and node E can be obtained through communicating with node $\mathrm{C}$.

\begin{tabular}{|c|c|c|c|c|c|c|c|}
\hline & G1 & G2 & A & B & C & D & E \\
\hline $\begin{array}{c}\text { Node } \\
\text { carbon } \\
\text { potential }\end{array}$ & known & known & known & known & known & known & known \\
\hline
\end{tabular}

According to the four iterations, the distribution of carbon emission flow of the system can be obtained at time $\mathrm{t}$. The calculation process of other time is same as this, due to the data interaction of computation units with time, the computation of carbon emission flow can't be interfered every times.

Distributedcalculation method of carbon emission flow on large-scale electric system

Distributed calculation process of carbon emission flow on large-scale electric system is similar to the simple system at the second part. According to the decision method of small world network,electric network generally have the characteristics of small-world network, any two nodescan be connected through a few middle nodes. This makes iterative calculation process of carbon emission flow completes within a few iterations. For large-scale electric system, it needs to verify whether it belongs to the small world network firstly, only small world network can adopt distributed calculate method of carbon emission flow, otherwise it will need more iterations to complete a computation.

\section{Method validation}

Electric systemof 24 nodespromulgated by the IEEE can be a example, The calculation effect is listed. The carbon potential of generator node is equal to the power density of carbon emissions, it is known. Iterative calculation process of other node carbon potential is shown in Table 1. U shows that node carbon potential has not been calculated in table, the carbon potential of all nodes can be calculated by 10 iterations, the calculation results and centralized computing results are the same. 
Table 1 Application on IEEE 24 nodes of distributed calculate method of carbon emission flow

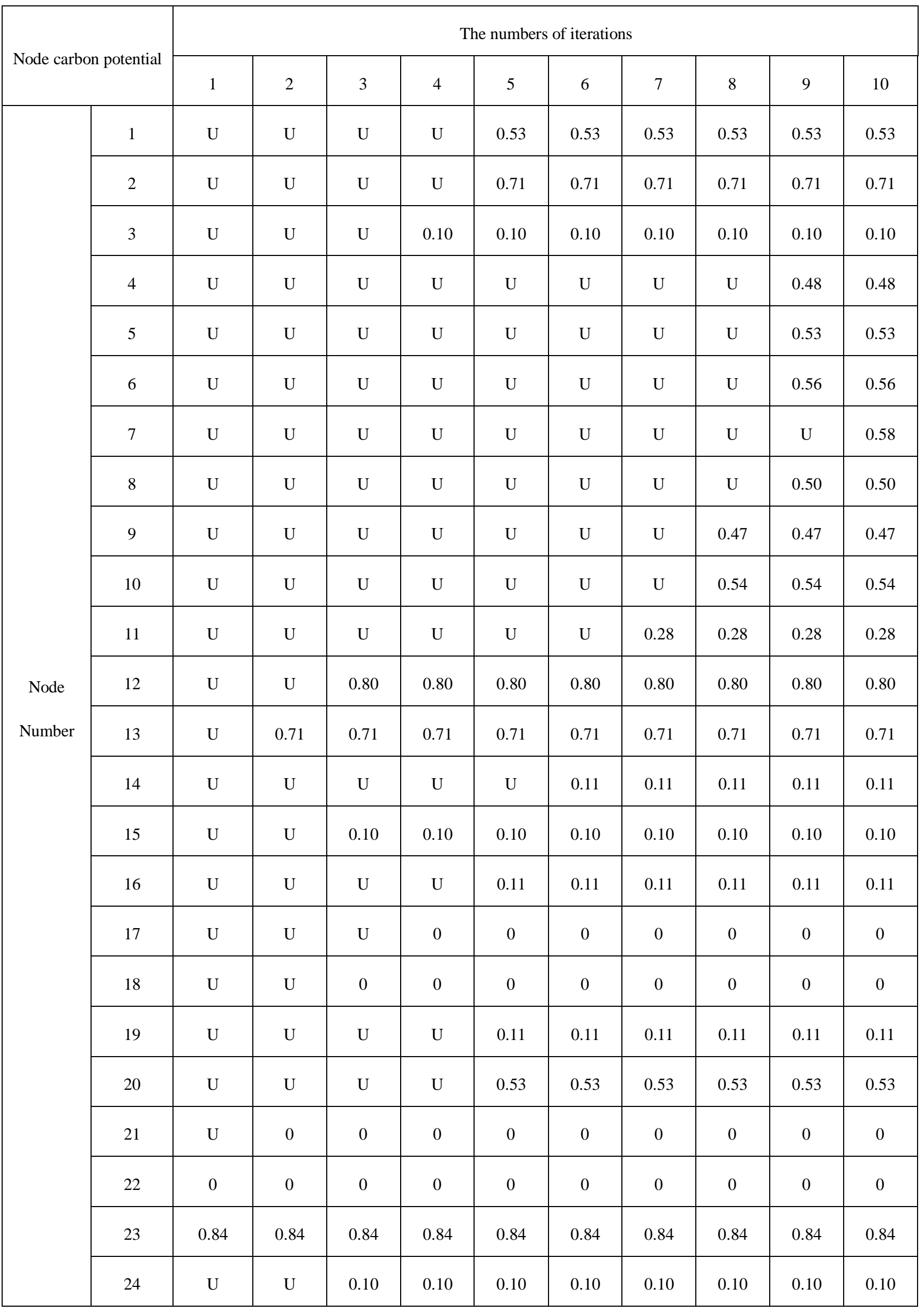


The distributed calculate method of carbon emission flow is applied on the larger electric system. The system has a total of 2277 nodes, nodeProportion of the carbon potential that can be calculated are shown in Fig. 4. The carbon potential of all nodes can be calculated by 15 iterations, the calculation results and centralized computing results are the same.

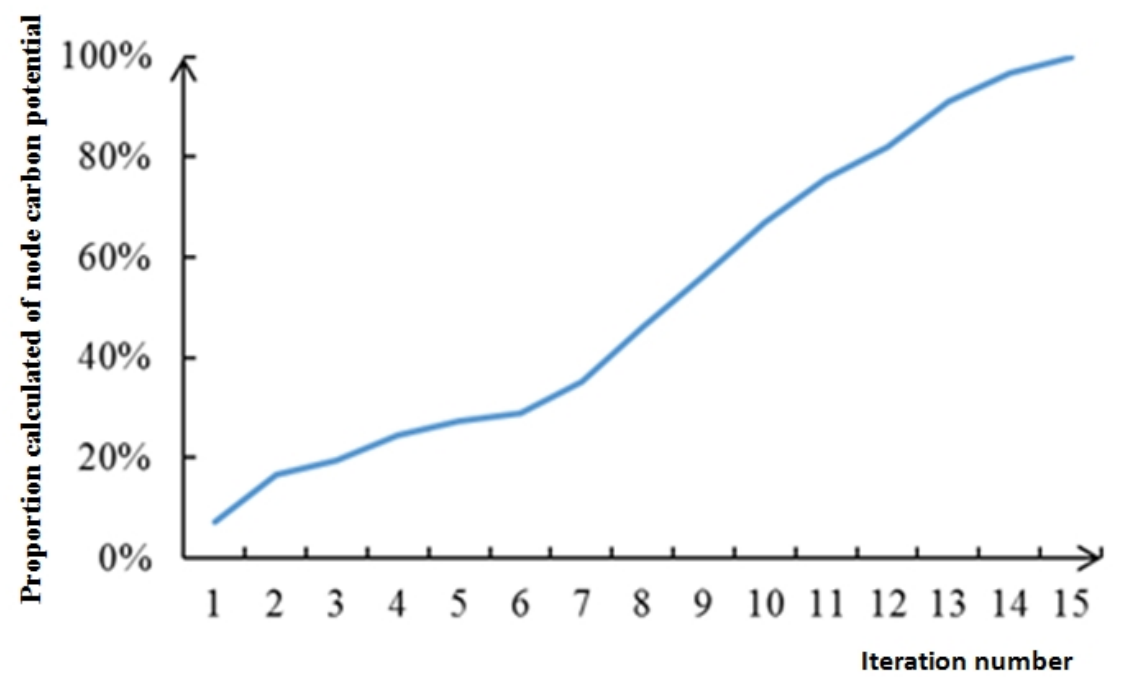

Fig. 4. The application effect of the present invention in large-scale electric system

\section{Conclusion}

Based on the calculation of carbon emission flow of electric system, this paper proposes a distributed computing method, which quickly solves the distribution of carbon emissions flow in the electric system. This method uses the concept of distributed computing, it reduces the computing burden of central server of the past the centralized calculation model of carbon emission flow, at the same time, adopting the distributed measurement unit also helps to improve the accuracy of measurement.

\section{References}

[1] Chen Qixin, Kang Chongqing, Xia Qing. Key Low-carbon Factors in the Evolution of Power Decarbonisation and Their Impacts on Generation Expansion Planning, J. Automation of Electric Power Systems, 2009, 33(15):18-23.

[2] Chen Qixin. Study on Theory and Method of Low Carbon Power System, D. Beijing, Tsinghua University, 2010.1.

[3] A Model for Generation Expansion Planning of Power System Based on Carbon Emission Trajectory Model under Low-Carbon Economy, J. Transactions of China Electrotechnical Society, 2011,25(11):175-181.

[4] Yuan C, Li F, Kuri B. Optimal Power Generation Mix towards an Emission Target. IEEE PES General Meeting, 2011:1-7.

[5] Zhou Tianrui, Kang Chongqing, Xu Qianyao, Chen Qixin. Preliminary Theoretical Investigation on Power System Carbon Emission Flow, J. Automation of Electric Power Systems, 2012, 07: 38-43+85.

[6] Lou Suhua, Lu Siyu, Wu Yaowu, Yin Xianggen. An Overview on Low-Carbon Power System Planning and Operation Optimization, J. Power System Technology, 2013, 06:1483-1490. 
[7] Liu Ye, Huang Guohe, Li Wei. Study on Dynamic Planning Model for the CO2 Emission Reduction of Power System Under Uncertainty Condition, J. Modern Electric Condition, 2012,05:66-71.

[8] Wang H, Nakata T. Analysis of the market penetration of clean coal technologies and its impacts in China's electricity sector. Energy Policy, 2009,37(1):338-351.

[9] Chen Q, Kang C, Xia Q, et al. Preliminary exploration on low-carbon technology roadmap of China's power sector. Energy, 2011,36(3):1500-1512. 